\title{
Correction: Electrocardiogram for heart rate evaluation during preterm resuscitation at birth: a randomized trial
}

Natalia V. Abbey, Vaishali Mashruwala, Heather M. Weydig, L. Steven Brown, Emma L. Ramon, John Ibrahim, Imran N. Mir, Myra H. Wyckoff and Vishal Kapadia

(c) The Author(s), under exclusive licence to the International Pediatric Research Foundation, Inc 2021

Pediatric Research (2022) 91:1624; https://doi.org/10.1038/s41390-021-01835-6

Correction to: Pediatric Research https://doi.org/10.1038/s41390021-01731-z, published online 13 October 2021
In this article the wrong table appeared as Table 2; the table should have appeared as shown below. The original article has been corrected.

Table 2. Resuscitation and delivery room characteristics.

\begin{tabular}{|c|c|c|c|}
\hline Characteristics & $\begin{array}{l}\text { ECG displayed } \\
(N=26)\end{array}$ & $\begin{array}{l}\text { Control } \\
(N=25)\end{array}$ & $P$ value \\
\hline Time to warmer & $22(17,25)$ & $18(16,23)$ & 0.67 \\
\hline Time to PO sensor placed & $29(17,37)$ & $31(17,51)$ & 0.88 \\
\hline Time to first visible HR & $64(52,85)(\mathrm{ECG})$ & $75(56,134)(P O)$ & 0.13 \\
\hline Time to ECG $H R \geq 100$ in bradycardic neonates, mean $\pm S D$ & $210 \pm 104$ & $211 \pm 107$ & 0.98 \\
\hline \multicolumn{4}{|l|}{$\mathrm{SpO}_{2}$ during resuscitation } \\
\hline Time to goal $\mathrm{SpO}_{2}$ from birth & $355(269,435)$ & $345(240,475)$ & 0.97 \\
\hline Time spent below goal $\mathrm{SpO}_{2}$ & $360(258,435)$ & $270(225,350)$ & 0.07 \\
\hline Time spent above goal $\mathrm{SpO}_{2}$ & $65(0,120)$ & $82(35,198)$ & 0.15 \\
\hline Positive pressure ventilation (PPV), $n(\%)$ & $17(65)$ & $19(76)$ & 0.60 \\
\hline Time to start PPV & $36(25,45)$ & $38(23,78)$ & 0.54 \\
\hline Total duration of PPV & $345(120,558)$ & $196(150,273)$ & 0.37 \\
\hline Delayed PPV, $n(\%)$ & $1(4)$ & $3(12)$ & 0.57 \\
\hline Continuous positive airway pressure only, $n(\%)$ & $9(35)$ & $6(24)$ & 0.60 \\
\hline Intubation, $n(\%)$ & $9(35)$ & $5(20)$ & 0.39 \\
\hline Chest compressions, $n(\%)$ & $0(0)$ & $0(0)$ & NS \\
\hline Rectal temperature after stabilization, mean $\pm S D$ & $37 \pm 0.6$ & $36.8 \pm 0.7$ & 0.87 \\
\hline Rectal temperature 36 to $36.5, n$ (\%) & $0(0)$ & $5(20)$ & NS \\
\hline Rectal temperature $<36, n(\%)$ & $2(8)$ & $0(0)$ & NS \\
\hline
\end{tabular}

Unless otherwise noted, data are presented as median (25th quartile, 75th quartile). All times are presented in seconds. All times are calculated from birth except the time to ECG leads placed, the time to PO sensor placed are calculated from the placement of infant in warmer. 